VARGAS PF; GALATTI FS; SOUZA JO; CASTOLDI R; CHARLO HCO; BRAZ LT. 2013. Physicochemical characteristics of experimental net melon hybrids developed in Brazil. Horticultura Brasileira 31: 351-355.

\title{
Physicochemical characteristics of experimental net melon hybrids developed in Brazil
}

\author{
Pablo F Vargasi'; Francine de S Galatti²; Jean de O Souza²; Renata Castoldi²; Hamilton Cesar de O \\ Charlo3; Leila T Braz \\ ${ }^{1}$ UNESP, Campus Registro, R. Nelson Brihi-Badur 430, Vila Tupy, 11900-000 Registro-SP; pablo@registro.unesp.br; ${ }^{2}$ UNESP-FCAV, \\ Rod. Prof. Paulo D. Castellane s/n, 14884-900 Jaboticabal-SP; ${ }^{3}$ Instituto Federal de Educação, Ciencia e Tecnologia do Triangulo \\ Mineiro, R. João Batista Ribeiro 4000, Distrito Industrial II, 38064-790 Uberaba-MG
}

\begin{abstract}
The objective of this study was to evaluate the physicochemical characteristics of parents and experimental hybrids of net melon improvement program developed in the Universidade Estadual Paulista (UNESP), in Jaboticabal, São Paulo state, Brazil. Six net melon lineages (Jab-3, Jab-7, Jab-11, Jab-18, Jab-9, and Jab-20), belonging to the breeding program of melon from the UNESP were used and all their hybrid combinations as well as their reciprocal crosses. We used the randomized blocks design, with three replications and 38 treatments. From these 38 treatments [six lines, 15 hybrids, 15 reciprocal crosses and two commercial cultivars (Bônus $n^{\circ} 2$ and Louis)] we evaluated following physicochemical characteristics: fruit mass, pulp thickness, fruit coat thickness, netting degree of the coat, seed looseness, soluble solids, titratable acidity, $\mathrm{pH}$, maturation index, pulp firmness, and Vitamin C. The minimum mass of $0.8 \mathrm{~kg}$ per fruit of net melon and the minimum of $10^{\circ}$ Brix soluble solids allowed to identify the genotypes: Jab-9 x Jab-3, Jab-18 x Jab-20, Jab-18 x Jab-11, Jab-7 x Jab-20, Jab-7 x Jab-11, Jab-20 x Jab-7, and, Jab-3 x Jab-20, as promising. These genotypes are therefore suitable for participating in assessment tests in the main net melon producing areas of Brazil and on different planting dates.
\end{abstract}

Keywords: Cucumis melo, plant breeding, fruit quality.

\section{RESUMO}

Características físico-químicas de frutos de híbridos experimentais de melão rendilhado

Objetivou-se com este estudo avaliar as características físico-químicas de parentais e híbridos experimentais de melão rendilhado, desenvolvidos na Universidade Estadual Paulista (UNESP), em Jaboticabal. Foram utilizadas seis linhagens (Jab-3; Jab-7; Jab-11; Jab-18; Jab-9 e Jab-20) pertencentes ao programa de melhoramento genético do meloeiro rendilhado da UNESP em Jaboticabal e, todas as combinações híbridas possíveis entre essas, inclusive os recíprocos. Foi utilizado o delineamento em blocos casualizados com três repetições. Dos 38 tratamentos, fizeram parte seis linhagens, 15 híbridos, 15 híbridos recíprocos e duas cultivares comerciais (Bônus $\mathrm{n}^{\circ} 2$ e Louis). Foram avaliadas massa do fruto, espessura da polpa, espessura da casca, rendilhamento da casca, desprendimento de semente, sólidos solúveis, acidez titulável, $\mathrm{pH}$, índice de maturação, firmeza da polpa, e, Vitamina C. A massa mínima de $0,8 \mathrm{~kg}$ por fruto de melão rendilhado e, sólidos solúveis de no mínimo $10^{\circ}$ Brix subsidiaram a identificação dos genótipos Jab-9 x Jab-3, Jab-18 x Jab-20, Jab-18 x Jab-11, Jab-7 x Jab-20, Jab-7 x Jab-11, Jab-20 x Jab-7, e Jab-3 x Jab-20 como promissores, devendo os mesmos serem avaliados nas principais regiões produtoras de melão rendilhado e em diferentes épocas de cultivo.

Palavras-chave: Cucumis melo, melhoramento de plantas, qualidade de fruto.

(Recebido para publicação em 28 de maio de 2012; aceito em 5 de junho de 2013) (Received on May 28, 2012; accepted on June 5, 2013)

$\mathrm{M}$ elon (Cucumis melo) is a very popular species all over the world. During the year 2008, Brazil produced 340,000 tons of this fruit (FAO, $2011)$ in an area of 16,000 hectares (Embrapa, 2011). Beyond the economic importance, melon production also has great social character, considering the generation of employment (Embrapa, 2011) of around 142,000.

Considering its nutritional value, melon is also very important due to its high content of pro-vitamins A ( $\beta$-carotene), vitamin $C$, vitamin $E$, folic acid and several nutrients (Gal et al., 2008).

Melon cultivation in Brazil is increasing steadily in recent years due to the growing acquisitive power of the Brazilian people and also because the export of melons from Brazil to countries in the northern hemisphere is favored due to the harvest season in Brazil takes place during the between- harvests period of those countries.

Among the noble classified melons, the net melon is one of the most outstanding because of its external appearance and its exquisite flavor. Paduan et al. (2007), in an analysis of several melon genotypes, found out the net melon to be superior to the others in terms of soluble solids and titratable acidity.

Despite the good perspectives, melon production chain is vulnerable 
because Brazil imports melon seeds (mainly from Japan and United States), that are expensive beyond increasing phytosanitary risks and the uncertainty of the availability of seeds when necessary (Vargas et al., 2010).

Having those problems in mind, the Universidade Estadual Paulista in Jaboticabal, São Paulo state, Brazil, started a program of genetic improvement of net melon in order to produce hybrid genotypes adapted to environmental conditions of Brazil. Therefore, the obtaining of superior lineages became the central focus of the program. In 2002, after several selection cycles, 20 superior lineages were selected. These were evaluated and grouped into dissimilarity groups (Mahalanobis general distance) or selected for agronomic characteristics viewing to identify divergent genitors to be used in hybridization projects.

Using hybrid plants in the production of vegetables presents a series of advantages such as the possibility of developing superior cultivars in a short period of time and assembling several important agronomic characteristics in one and the same genotype.

Having those considerations in mind, the objective of this research was to evaluate physicochemical characteristics of parent lines and experimental hybrids of net melon.

\section{MATERIAL AND METHODS}

The project was conducted at the Universidade Estadual Paulista, in Jaboticabal, São Paulo state, Brazil $\left(21^{\circ} 15^{\prime} 22^{\prime \prime} \mathrm{S}, 48^{\circ} 18^{\prime} 58^{\prime \prime} \mathrm{W}\right.$, altitude of $575 \mathrm{~m})$. The climate, according to the classification of Köppen is Aw type with a transition to Cwa. Two $50 \mathrm{~m}$ long, $6 \mathrm{~m}$ wide and $3 \mathrm{~m}$ high greenhouses were used for the observations. The greenhouses were covered with a low density $150 \mu \mathrm{m}$ thick polyethylene film.

Net melon lines Jab-3, Jab-7, Jab-11, Jab-18, Jab-9, and Jab-20 and all their possible hybrid combinations as well as all the reciprocal crosses were submitted to evaluations.

The experimental units used a randomized blocks design, with 38 treatments (six lines, 15 hybrids of their reciprocal crosses and two commercial cultivars (Bônus n ${ }^{\circ} 2$ and Louis). In each plot, five plants from two central rows were evaluated for all characteristics.

The genotypes were sown on August 1, 2007, in expanded polyester trays with 128 cells filled with Plantmax HT $^{\circledR}$ (a special substratum for vegetables). Seedlings were transplanted when they presented the first fully expanded non cotiledonary leaf to polyethylene $13 \mathrm{~L}$ vases filled with coconut husk fiber as a substratum. Each vase received one seedling and the vases were arranged in rows spaced of $1.0 \times 0.5 \mathrm{~m}$.

The surface of the soil in each vase was covered with sugar cane bagasse and the vases were placed on wood balconies so to stay above the greenhouse pavement.

The plants were fertigated with a solution containing in 1,000 liters, as recommended by Castellane \& Araujo (1994), $805 \mathrm{~g}$ of calcium nitrate, $277 \mathrm{~g}$ of potassium nitrate, $238 \mathrm{~g}$ of potassium chloride, $155 \mathrm{~g}$ of mono-ammonium phosphate, $240 \mathrm{~g}$ of magnesium sulphate, $36.6 \mathrm{~g}$ of Tenso-Iron, 2.544 $\mathrm{g}$ of manganese sulphate, $1.90 \mathrm{~g}$ of borax, $1.15 \mathrm{~g}$ of zinc sulphate, $0.12 \mathrm{~g}$ of copper sulphate, and $0.12 \mathrm{~g}$ of sodium molybdate.

Fertigation was automated with the help of a timer. It started at 7 am and ended at $6 \mathrm{pm}$. When the plants were in their initial stage of development, each fertigation cycle was planned to last 5 minutes every hour. After the plants had reached the mature stage the cycle was of 15 minutes of fertigation every hour. Fertigation rate was of $14 \mathrm{~mL} \mathrm{~min}^{-1}$.

Plants were staked using plastic threads tied to localized wires close to the vase, $2 \mathrm{~m}$ above the greenhouse pavement. When the plants reached their maximum height, the apical buds were pruned allowing only one stem per plant. Bud pruning was made up to the $10^{\text {th }}$ internode. Subsequently, lateral buds were left on the plant and, after fruit onset, bud pruning was continued.

Diseases and insects were preventively controlled according to the plants necessity. The products Acetamiprido, Thiacloprid, and
Thiamethoxam were used for the control of the white fly, Clorotalonil + Tiofanato Metílico, Iprodiona, and Difenoconazole for the control of gummy stem blight, Azoxystrobin, Tiofanato Metílico, and Fenarimol for the control of oidium, Deltametrina and Lambdacyhalothrin for the control of pickleworm, Triclorfom for the control of corn earworm, Clorotalonil, Clorotalonil + Tiofanato Metílico and Clorothalonil + Oxicloreto copper for the control of mildew, and Tiofanato Metilico + Chlorothalonil and Clorothalonil + Oxicloreto copper for the control of cercosporiosis. Pollination was made by bees.

Two fruits per plant were maintained. Pruning was made after fruit onset. Harvesting point was determined by the development of the abscission layer near the peduncle or by the changing of coat color. Plants not bearing two fruits were discarded.

The characteristics evaluated were: 1) fruit mass (fruit mass obtained on each plot; 2) pulp thickness (measured with a digital caliper, in $\mathrm{mm}$ ); 3) fruit coat thickness (measured with a digital caliper); 4) netting degree of the coat [visually determined according to procedure described by Rizzo \& Braz (2004), classified as 1 (weak), 2 (medium), and 3 (strong)]; 5) seed looseness [classified as $1=$ difficult, $2=$ intermediate, and 3= easy (a dessert spoon was used to loose the seeds)]; 6) soluble solids (measured by a digital refractometer adjusted to $20^{\circ} \mathrm{C}$, expressed in ${ }^{\circ} \mathrm{Brix}$ ); 7) titratable acidity [determined in a $10 \mathrm{~mL}$ volume of fruit juice to which $40 \mathrm{~mL}$ of distilled water and three drops of a $1 \%$ solution of alcoholic phenolphthalein were added; titration was made with a $0.1 \mathrm{~N} \mathrm{NaOH}$ solution up to the turning point; the data were expressed in \% of citric acid (Instituto Adolfo Lutz, 1985)]; pH was determined in the juice extract with the help of a digital pHmeter; maturation index was given by the relation between solid solubles and titratable acidity (SS/ AT); pulp firmness was evaluated by a digital penetrometer pressed against two different but equidistant regions of the fruit (Newton); Vitamin $\mathrm{C}$ as resulting from the titration with 2,6 sodium 
Table 1. Mean values of five fruit characteristics of 38 net melon genotypes (valores médios de cinco características de frutos de 38 genótipos de melão rendilhado). Jaboticabal, UNESP, 2012.

\begin{tabular}{|c|c|c|c|c|c|c|c|c|c|c|c|}
\hline \multicolumn{2}{|c|}{ Genotypes } & \multicolumn{2}{|l|}{$\begin{array}{c}\text { Mass } \\
(\mathrm{kg})\end{array}$} & \multicolumn{2}{|c|}{$\begin{array}{c}\mathbf{E P}^{1} \\
(\mathrm{~mm})\end{array}$} & \multicolumn{2}{|c|}{$\begin{array}{c}\mathrm{EC} \\
(\mathrm{mm})\end{array}$} & \multicolumn{2}{|c|}{$\begin{array}{c}\text { RC } \\
\text { (grade) }\end{array}$} & \multicolumn{2}{|c|}{$\begin{array}{c}\text { DS } \\
\text { (grade) }\end{array}$} \\
\hline Jab-9 & x Jab-9 & 0.962 & $b^{2}$ & 31.48 & $\mathrm{~b}$ & 1.58 & $\mathrm{a}$ & 2.83 & $\mathrm{a}$ & 2.55 & $\mathrm{~b}$ \\
\hline Jab-9 & x Jab-18 & 0.879 & $\mathrm{c}$ & 32.33 & $\mathrm{~b}$ & 1.64 & $\mathrm{a}$ & 3.00 & $\mathrm{a}$ & 2.66 & $\mathrm{a}$ \\
\hline Jab-9 & x Jab-7 & 0.689 & $\mathrm{e}$ & 29.18 & $\mathrm{c}$ & 1.61 & $\mathrm{a}$ & 2.31 & $\mathrm{~b}$ & 2.05 & $\mathrm{c}$ \\
\hline Jab-9 & x Jab-20 & 1.112 & $\mathrm{a}$ & 30.04 & $\mathrm{c}$ & 1.43 & $\mathrm{a}$ & 2.36 & $\mathrm{~b}$ & 3.00 & $\mathrm{a}$ \\
\hline Jab-9 & x Jab-11 & 0.780 & $\mathrm{~d}$ & 31.15 & $\mathrm{~b}$ & 1.53 & $\mathrm{a}$ & 2.62 & $\mathrm{~b}$ & 3.00 & $\mathrm{a}$ \\
\hline Jab-9 & x Jab-3 & 0.932 & $\mathrm{~b}$ & 31.88 & $\mathrm{~b}$ & 1.51 & $\mathrm{a}$ & 2.75 & $\mathrm{a}$ & 2.72 & $\mathrm{a}$ \\
\hline Jab-18 & x Jab-9 & 0.645 & e & 31.87 & $\mathrm{~b}$ & 1.66 & $\mathrm{a}$ & 2.67 & $\mathrm{a}$ & 2.07 & $\mathrm{c}$ \\
\hline Jab-18 & x Jab-18 & 0.959 & $\mathrm{~b}$ & 30.20 & $\mathrm{c}$ & 1.49 & $\mathrm{a}$ & 2.83 & $\mathrm{a}$ & 3.00 & $\mathrm{a}$ \\
\hline Jab-18 & x Jab-7 & 0.978 & $\mathrm{~b}$ & 32.50 & $\mathrm{~b}$ & 1.47 & $\mathrm{a}$ & 3.00 & $\mathrm{a}$ & 2.25 & $\mathrm{~b}$ \\
\hline Jab-18 & x Jab-20 & 0.860 & $\mathrm{c}$ & 31.17 & $\mathrm{~b}$ & 1.69 & $\mathrm{a}$ & 3.00 & $\mathrm{a}$ & 2.33 & $b$ \\
\hline Jab-18 & x Jab-11 & 0.907 & $\mathrm{c}$ & 32.70 & $b$ & 1.46 & $\mathrm{a}$ & 2.67 & $\mathrm{a}$ & 2.67 & $\mathrm{a}$ \\
\hline Jab-18 & x Jab-3 & 0.834 & d & 31.79 & $\mathrm{~b}$ & 1.45 & $\mathrm{a}$ & 2.89 & $\mathrm{a}$ & 2.30 & $b$ \\
\hline Jab-7 & x Jab-9 & 0.705 & e & 28.04 & $\mathrm{c}$ & 1.43 & $\mathrm{a}$ & 2.53 & b & 1.87 & $\mathrm{c}$ \\
\hline Jab-7 & x Jab-18 & 0.773 & $\mathrm{~d}$ & 28.35 & $\mathrm{c}$ & 1.71 & $\mathrm{a}$ & 3.00 & $\mathrm{a}$ & 2.44 & $\mathrm{~b}$ \\
\hline Jab-7 & x Jab-7 & 0.568 & $\mathrm{f}$ & 26.38 & $\mathrm{c}$ & 1.64 & $\mathrm{a}$ & 2.89 & $\mathrm{a}$ & 1.59 & $\mathrm{c}$ \\
\hline Jab-7 & x Jab-20 & 0.953 & $\mathrm{~b}$ & 32.29 & $\mathrm{~b}$ & 1.58 & $\mathrm{a}$ & 2.44 & $\mathrm{~b}$ & 1.77 & $\mathrm{c}$ \\
\hline Jab-7 & x Jab-11 & 0.939 & $\mathrm{~b}$ & 31.57 & $\mathrm{~b}$ & 1.48 & $\mathrm{a}$ & 2.08 & $b$ & 1.59 & $\mathrm{c}$ \\
\hline Jab-7 & x Jab-3 & 0.694 & e & 30.50 & $\mathrm{c}$ & 1.71 & $\mathrm{a}$ & 2.87 & $\mathrm{a}$ & 1.64 & $\mathrm{c}$ \\
\hline Jab-20 & x Jab-9 & 0.977 & $\mathrm{~b}$ & 31.27 & $\mathrm{~b}$ & 1.46 & $\mathrm{a}$ & 2.61 & $\mathrm{~b}$ & 2.17 & $b$ \\
\hline Jab-20 & x Jab-18 & 0.785 & $\mathrm{~d}$ & 32.33 & $b$ & 1.53 & $\mathrm{a}$ & 3.00 & $\mathrm{a}$ & 2.61 & b \\
\hline Jab-20 & x Jab-7 & 0.833 & $\mathrm{~d}$ & 29.42 & $\mathrm{c}$ & 1.56 & $\mathrm{a}$ & 2.89 & $\mathrm{a}$ & 2.44 & b \\
\hline Jab-20 & x Jab-20 & 0.987 & $\mathrm{~b}$ & 31.29 & $\mathrm{~b}$ & 1.48 & $\mathrm{a}$ & 2.48 & $\mathrm{~b}$ & 2.22 & $\mathrm{~b}$ \\
\hline Jab-20 & x Jab-11 & 1.034 & $\mathrm{a}$ & 31.15 & $\mathrm{~b}$ & 2.57 & $\mathrm{a}$ & 2.06 & $\mathrm{~b}$ & 2.28 & $b$ \\
\hline Jab-20 & x Jab-3 & 1.044 & $\mathrm{a}$ & 30.40 & $\mathrm{c}$ & 1.64 & $\mathrm{a}$ & 3.00 & $\mathrm{a}$ & 3.00 & $\mathrm{a}$ \\
\hline Jab-11 & x Jab-9 & 0.960 & $\mathrm{~b}$ & 28.97 & $\mathrm{c}$ & 1.49 & $\mathrm{a}$ & 2.83 & $\mathrm{a}$ & 2.58 & $b$ \\
\hline Jab-11 & x Jab-18 & 1.017 & $\mathrm{a}$ & 30.08 & $\mathrm{c}$ & 1.62 & $\mathrm{a}$ & 2.77 & $\mathrm{a}$ & 3.00 & $\mathrm{a}$ \\
\hline Jab-11 & x Jab-7 & 0.741 & $\mathrm{~d}$ & 29.73 & $\mathrm{c}$ & 1.57 & $\mathrm{a}$ & 2.45 & $\mathrm{~b}$ & 2.22 & b \\
\hline Jab-11 & x Jab-20 & 1.084 & $\mathrm{a}$ & 31.42 & $\mathrm{~b}$ & 1.58 & $\mathrm{a}$ & 2.50 & $\mathrm{~b}$ & 2.58 & $b$ \\
\hline Jab-11 & x Jab-11 & 0.932 & $\mathrm{~b}$ & 31.69 & $\mathrm{~b}$ & 1.43 & $\mathrm{a}$ & 2.80 & $\mathrm{a}$ & 2.75 & $\mathrm{a}$ \\
\hline Jab-11 & x Jab-3 & 0.890 & $\mathrm{c}$ & 32.65 & $\mathrm{~b}$ & 1.49 & $\mathrm{a}$ & 2.67 & $\mathrm{a}$ & 2.39 & b \\
\hline Jab-3 & x Jab-9 & 0.936 & $\mathrm{~b}$ & 29.88 & $\mathrm{c}$ & 1.54 & $\mathrm{a}$ & 3.00 & $\mathrm{a}$ & 3.00 & $\mathrm{a}$ \\
\hline Jab-3 & x Jab-18 & 0.689 & e & 29.90 & $\mathrm{c}$ & 1.39 & $\mathrm{a}$ & 2.61 & $\mathrm{~b}$ & 2.53 & b \\
\hline Jab-3 & x Jab-7 & 0.707 & e & 31.25 & $\mathrm{~b}$ & 1.35 & $\mathrm{a}$ & 2.83 & $\mathrm{a}$ & 1.39 & d \\
\hline Jab-3 & x Jab-20 & 0.896 & $\mathrm{c}$ & 30.23 & $\mathrm{c}$ & 1.55 & $\mathrm{a}$ & 2.72 & $\mathrm{a}$ & 2.22 & $b$ \\
\hline Jab-3 & x Jab-11 & 0.979 & $\mathrm{~b}$ & 32.92 & $\mathrm{~b}$ & 1.69 & $\mathrm{a}$ & 2.78 & $\mathrm{a}$ & 2.55 & b \\
\hline Jab-3 & x Jab-3 & 0.801 & $\mathrm{~d}$ & 29.84 & $\mathrm{c}$ & 1.41 & $\mathrm{a}$ & 3.00 & a & 2.67 & $\mathrm{a}$ \\
\hline Bônus & $n^{\circ} 2$ & 0.994 & $\mathrm{~b}$ & 35.89 & $\mathrm{a}$ & 1.60 & $\mathrm{a}$ & 2.75 & $\mathrm{a}$ & 1.00 & d \\
\hline Louis & & 1.082 & $\mathrm{a}$ & 35.32 & $\mathrm{a}$ & 1.59 & $\mathrm{a}$ & 2.44 & $\mathrm{~b}$ & 1.33 & d \\
\hline Test F & & $20,66^{* *}$ & & $3.92 *$ & & 0.9 & & 2.58 & & $6.40 * *$ & \\
\hline $\mathrm{CV}(\%)$ & & 5.84 & & 5.05 & & 21.3 & & 10.03 & & 15.05 & \\
\hline
\end{tabular}

${ }^{1} \mathrm{EP}=$ pulp thickness; $\mathrm{EC}=$ fruit coat thickness; $\mathrm{RC}=$ netting degree of the coat $(1=$ weak, $2=$ medium, $3=$ strong $)$; DS $=$ seed looseness $(1=$ difficult, $2=$ intermediate, $3=$ easy $) ;{ }^{2}$ Means in the same column, followed by the same letter, are not significantly different (Scott Knott, $5 \%$ ); ns= not significant (Scott Knott, 5\%);**= significant (Scott Knott, 1\%); [EP= espessura de polpa; $\mathrm{EC}=$ espessura da casca; $\mathrm{RC}=$ rendilhamento de casca $(1=$ fraco, $2=$ médio, $3=$ intenso); $\mathrm{DS}=$ desprendimento de sementes (1= difícil, 2= intermediário, $3=$ fácil); Médias seguidas da mesma letra, na coluna, não diferem entre si (Scott Knott, 5\%); ns= não significativo (Scott Knott, 5\%); **= significativo (Scott Knott, 1\%)]. dichlorophenol indophenol (2,6 DINa) and expressed in $\mathrm{mg}$ of ascorbic acid per $100 \mathrm{~mL}$ of juice.

For each one of the evaluated characteristics, the F test was applied and, when significant, the means were compared by the Scott Knott test at the $5 \%$ level of significance (Cruz, 2006).

\section{RESULTS AND DISCUSSION}

Fruit mass varied between 0.57 and $1.11 \mathrm{~kg}$. The genotypes Jab-9 x Jab20, Jab-20 x Jab-11, Jab-20 x Jab-3, Jab-11 x Jab-18, Jab-11 x Jab-20 and Louis showed the highest fruit masses, significantly superior to the fruit masses of the other genotypes (Table 1). The values found in this work were higher than those reported by Rizzo \& Braz $(2001,2004)$ which were obtained in soil cultivation under protected environment. On the other hand, Costa et al. (2004), in a hydroponic cultivation, reported results similar to the ones of the present work.

The genotypes 'Bônus $\mathrm{n}^{\circ} 2$ ' and 'Louis', which are considered as standard, were those with the thickest pulp (Table 1), with values were similar to those reported by Vargas et al. (2008a). Genotypes Jab-20 x Jab-11 and their reciprocal cross, although part of a second group, had also a reasonably thick pulp as well as high fruit mass, as mentioned earlier.

Genotypes Jab-9 x Jab-18, Jab-18 x Jab-7, Jab-18 x Jab-20, Jab-7 x Jab-18, Jab-20 x Jab-18, Jab-20 x Jab-3, Jab-3 x Jab-9, and Jab-3 x Jab-3 had the highest coat netting degree (grade 3) although they did not differ significantly from genotypes Jab-9 x Jab-9, Jab-9 x Jab-3, Jab-18 x Jab-9, Jab-18 x Jab-18, Jab-18 x Jab-11, Jab-18 x Jab-3, Jab-7 x Jab-7, Jab-7 x Jab-3, Jab-20 x Jab-7, Jab-11 x Jab- 9, Jab-11 x Jab-18, Jab-11 x Jab-11, Jab-11 x Jab-3, Jab-3 x Jab-7, Jab-3 x Jab-20, Jab-3 x Jab-11, and Bônus n ${ }^{\circ} 2$ (Table 1). The external appearance of net melon fruits is a quality attribute and, commercially it is desirable that melons present high intensity of netting of the coat, making them attractive to the consumer at the time of acquisition.

Seed looseness and the pulp placenta were more adherent in genotypes 
'Bônus n²', 'Louis' and Jab-3 x Jab-7, whose values significantly differed from those shown by the other genotypes. Seed looseness was highest in genotypes Jab-9 x Jab-18, Jab-9 x Jab-20, Jab-9 x
Jab-11, Jab-9 x Jab-3, Jab-18 x Jab-18, Jab-18 x Jab-11, Jab-20 x Jab-3, Jab11 x Jab-18, Jab-11 x Jab-11, Jab-3 x Jab-9, and Jab-3 x Jab-3. Seeds and placenta with a high degree of looseness is a negative factor since they may be loosened during transportation and this may be a factor of internal fermentation which causes reduction in the shelf life of the fruits.

Table 2. Mean values of six fruit characteristics of 38 net melon genotypes (valores médios de seis características de frutos de 38 genótipos de melão rendilhado). Jaboticabal, UNESP, 2012.

\begin{tabular}{|c|c|c|c|c|c|c|c|}
\hline \multicolumn{2}{|c|}{ Genotypes } & \multirow{2}{*}{$\frac{\mathbf{S S}^{\mathbf{1}}\left({ }^{\mathbf{o}} \mathbf{B r i x}\right)}{8.99 \mathrm{~d}^{2}}$} & \multirow{2}{*}{$\begin{array}{l}\text { AT (\%) } \\
0.073 \mathrm{c}\end{array}$} & \multirow{2}{*}{$\frac{\text { Ratio SS/AT }}{120.14 \mathrm{c}}$} & \multirow{2}{*}{$\frac{\mathbf{p H}}{7.69 \mathrm{a}}$} & \multirow{2}{*}{$\frac{\text { VitC (mg) }}{22.75 \mathrm{a}}$} & \multirow{2}{*}{$\frac{\text { Pulp firmness (N) }}{13.05 \mathrm{c}}$} \\
\hline Jab-9 & x Jab-9 & & & & & & \\
\hline Jab-9 & x Jab-18 & $10.95 \mathrm{~b}$ & $0.087 \mathrm{~b}$ & $132.48 \mathrm{c}$ & $7.08 \mathrm{a}$ & $7.70 \mathrm{~d}$ & $15.02 \mathrm{c}$ \\
\hline Jab-9 & x Jab-7 & $13.03 \mathrm{a}$ & $0.093 \mathrm{~b}$ & $130.60 \mathrm{c}$ & $7.45 \mathrm{a}$ & $15.44 \mathrm{c}$ & $15.99 \mathrm{~b}$ \\
\hline Jab-9 & x Jab-20 & $8.75 \mathrm{~d}$ & $0.070 \mathrm{c}$ & $125.80 \mathrm{c}$ & $7.39 \mathrm{a}$ & $15.60 \mathrm{c}$ & $13.25 \mathrm{c}$ \\
\hline Jab-9 & x Jab-11 & $10.13 \mathrm{c}$ & $0.087 \mathrm{~b}$ & $101.97 \mathrm{c}$ & $7.82 \mathrm{a}$ & $17.92 \mathrm{~b}$ & $15.02 \mathrm{c}$ \\
\hline Jab-9 & x Jab-3 & $11.85 \mathrm{a}$ & $0.083 \mathrm{~b}$ & $141.78 \mathrm{~b}$ & $7.38 \mathrm{a}$ & $11.94 \mathrm{c}$ & $12.33 \mathrm{~d}$ \\
\hline Jab-18 & x Jab-9 & $11.71 \mathrm{a}$ & $0.093 \mathrm{~b}$ & $121.14 \mathrm{c}$ & $7.40 \mathrm{a}$ & $14.15 \mathrm{c}$ & $14.95 \mathrm{c}$ \\
\hline Jab-18 & x Jab-18 & $8.77 \mathrm{~d}$ & $0.087 \mathrm{~b}$ & $113.88 \mathrm{c}$ & $7.00 \mathrm{a}$ & $6.26 \mathrm{~d}$ & $11.32 \mathrm{~d}$ \\
\hline Jab-18 & x Jab-7 & $10.81 \mathrm{~b}$ & $0.077 \mathrm{c}$ & $139.08 \mathrm{~b}$ & $7.49 \mathrm{a}$ & $7.83 \mathrm{~d}$ & $16.19 \mathrm{~b}$ \\
\hline Jab-18 & x Jab-20 & $11.61 \mathrm{a}$ & $0.090 \mathrm{~b}$ & $127.00 \mathrm{c}$ & $7.20 \mathrm{a}$ & $5.68 \mathrm{~d}$ & $16.87 \mathrm{~b}$ \\
\hline Jab-18 & x Jab-11 & $11.59 \mathrm{a}$ & $0.087 \mathrm{~b}$ & $125.77 \mathrm{c}$ & $7.19 \mathrm{a}$ & $23.35 \mathrm{a}$ & $14.51 \mathrm{c}$ \\
\hline Jab-18 & x Jab-3 & $11.24 \mathrm{~b}$ & $0.080 \mathrm{c}$ & $140.16 \mathrm{~b}$ & $7.28 \mathrm{a}$ & $6.73 \mathrm{~d}$ & $17.94 \mathrm{~b}$ \\
\hline Jab-7 & x Jab-9 & $11.63 \mathrm{a}$ & $0.103 \mathrm{a}$ & $118.18 \mathrm{c}$ & $7.43 \mathrm{a}$ & $7.18 \mathrm{~d}$ & $16.02 \mathrm{~b}$ \\
\hline Jab-7 & x Jab-18 & $11.94 \mathrm{a}$ & $0.090 \mathrm{~b}$ & $133.31 \mathrm{c}$ & $7.20 \mathrm{a}$ & $6.88 \mathrm{~d}$ & $15.76 \mathrm{~b}$ \\
\hline Jab-7 & x Jab-7 & $11.28 \mathrm{~b}$ & $0.080 \mathrm{c}$ & $167.43 \mathrm{a}$ & $7.64 \mathrm{a}$ & $24.53 \mathrm{a}$ & $22.68 \mathrm{a}$ \\
\hline Jab-7 & x Jab-20 & $12.28 \mathrm{a}$ & $0.080 \mathrm{c}$ & $142.01 \mathrm{~b}$ & $7.67 \mathrm{a}$ & $23.88 \mathrm{a}$ & $16.59 \mathrm{~b}$ \\
\hline Jab-7 & x Jab-11 & $11.29 \mathrm{~b}$ & $0.083 \mathrm{~b}$ & $129.42 \mathrm{c}$ & $7.67 \mathrm{a}$ & $24.08 \mathrm{a}$ & $18.58 \mathrm{a}$ \\
\hline Jab-7 & x Jab-3 & $11.51 \mathrm{a}$ & $0.090 \mathrm{~b}$ & $121.86 \mathrm{c}$ & $7.53 \mathrm{a}$ & $19.03 \mathrm{~b}$ & $21.00 \mathrm{a}$ \\
\hline Jab-20 & x Jab-9 & $9.29 \mathrm{~d}$ & $0.077 \mathrm{c}$ & $116.68 \mathrm{c}$ & $7.58 \mathrm{a}$ & $26.83 \mathrm{a}$ & $13.34 \mathrm{c}$ \\
\hline Jab-20 & x Jab-18 & $11.14 \mathrm{~b}$ & $0.090 \mathrm{~b}$ & $118.91 \mathrm{c}$ & $7.21 \mathrm{a}$ & $8.86 \mathrm{~d}$ & $16.32 \mathrm{~b}$ \\
\hline Jab-20 & x Jab-7 & $10.70 \mathrm{~b}$ & $0.080 \mathrm{c}$ & $119.66 \mathrm{c}$ & $6.78 \mathrm{a}$ & $23.15 \mathrm{a}$ & $18.10 \mathrm{~b}$ \\
\hline Jab-20 & x Jab-20 & $8.65 \mathrm{~d}$ & $0.073 \mathrm{c}$ & $118.35 \mathrm{c}$ & $7.73 \mathrm{a}$ & $28.42 \mathrm{a}$ & $14.03 \mathrm{c}$ \\
\hline Jab-20 & x Jab-11 & $9.37 \mathrm{~d}$ & $0.083 \mathrm{~b}$ & $107.16 \mathrm{c}$ & $7.65 \mathrm{a}$ & $25.53 \mathrm{a}$ & $14.48 \mathrm{c}$ \\
\hline Jab-20 & x Jab-3 & $9.95 \mathrm{c}$ & $0.060 \mathrm{c}$ & $160.32 \mathrm{a}$ & $7.52 \mathrm{a}$ & $20.04 \mathrm{~b}$ & $12.08 \mathrm{~d}$ \\
\hline Jab-11 & x Jab-9 & $10.58 \mathrm{~b}$ & $0.073 \mathrm{c}$ & $141.93 \mathrm{~b}$ & $7.69 \mathrm{a}$ & $17.79 \mathrm{~b}$ & $15.52 \mathrm{~b}$ \\
\hline Jab-11 & x Jab-18 & $10.87 \mathrm{~b}$ & $0.090 \mathrm{~b}$ & $123.75 \mathrm{c}$ & $7.13 \mathrm{a}$ & $10.67 \mathrm{c}$ & $15.86 \mathrm{~b}$ \\
\hline Jab-11 & x Jab-7 & $11.05 \mathrm{~b}$ & $0.077 \mathrm{c}$ & $156.88 \mathrm{a}$ & $7.61 \mathrm{a}$ & $16.53 \mathrm{~b}$ & $19.18 \mathrm{a}$ \\
\hline Jab-11 & x Jab-20 & $8.20 \mathrm{~d}$ & $0.077 \mathrm{c}$ & $104.31 \mathrm{c}$ & $7.83 \mathrm{a}$ & $25.07 \mathrm{a}$ & $13.92 \mathrm{c}$ \\
\hline Jab-11 & x Jab-11 & $9.38 \mathrm{~d}$ & $0.070 \mathrm{c}$ & $126.36 \mathrm{c}$ & $7.62 \mathrm{a}$ & $27.17 \mathrm{a}$ & $15.48 \mathrm{~b}$ \\
\hline Jab-11 & x Jab-3 & $10.27 \mathrm{c}$ & $0.070 \mathrm{c}$ & $143.80 \mathrm{~b}$ & $7.36 \mathrm{a}$ & $11.86 \mathrm{c}$ & $9.47 \mathrm{~d}$ \\
\hline Jab-3 & x Jab-9 & $9.82 \mathrm{c}$ & $0.090 \mathrm{~b}$ & $109.16 \mathrm{c}$ & $7.90 \mathrm{a}$ & $26.54 \mathrm{a}$ & $12.15 \mathrm{~d}$ \\
\hline Jab-3 & x Jab-18 & $10.97 \mathrm{~b}$ & $0.083 \mathrm{~b}$ & $130.19 \mathrm{c}$ & $7.21 \mathrm{a}$ & $13.81 \mathrm{c}$ & $16.81 \mathrm{~b}$ \\
\hline Jab-3 & x Jab-7 & $10.32 \mathrm{c}$ & $0.080 \mathrm{c}$ & $159.51 \mathrm{a}$ & $7.34 \mathrm{a}$ & $8.98 \mathrm{~d}$ & $19.90 \mathrm{a}$ \\
\hline Jab-3 & x Jab-20 & $10.58 \mathrm{~b}$ & $0.073 \mathrm{c}$ & $136.67 \mathrm{~b}$ & $7.77 \mathrm{a}$ & $24.89 \mathrm{a}$ & $11.80 \mathrm{~d}$ \\
\hline Jab-3 & x Jab-11 & $10.84 \mathrm{~b}$ & $0.063 \mathrm{c}$ & $140.77 \mathrm{~b}$ & $7.55 \mathrm{a}$ & $18.99 \mathrm{~b}$ & $12.58 \mathrm{~d}$ \\
\hline Jab-3 & x Jab-3 & $10.53 \mathrm{~b}$ & $0.080 \mathrm{c}$ & $160.66 \mathrm{a}$ & $7.47 \mathrm{a}$ & $16.99 \mathrm{~b}$ & $9.45 \mathrm{~d}$ \\
\hline Bônus $n$ & $n^{\circ} 2$ & $12.27 \mathrm{a}$ & $0.090 \mathrm{~b}$ & $130.39 \mathrm{c}$ & $7.19 \mathrm{a}$ & $13.41 \mathrm{c}$ & $17.27 \mathrm{~b}$ \\
\hline Louis & & $11.31 \mathrm{~b}$ & $0.110 \mathrm{a}$ & $107.70 \mathrm{c}$ & $6.85 \mathrm{a}$ & $5.68 \mathrm{~d}$ & $19.34 \mathrm{a}$ \\
\hline Test F & & $10.46 * *$ & $4.48 * *$ & $3.64 * *$ & $1.60 *$ & $2.30 * *$ & $7.24 * *$ \\
\hline CV $(\%)$ & & 5.69 & 10.00 & 11.46 & 4.99 & 16.23 & 12.49 \\
\hline
\end{tabular}

${ }^{1} \mathrm{SS}=$ solid solubles $\left({ }^{\circ} \mathrm{Brix}\right) ; \mathrm{AT}=$ titratable acidity $(\%$ of citric acid $) ; \mathrm{VitC}=$ vitamin $\mathrm{C}(\mathrm{mg}$ of ascorbic acid $/ 100 \mathrm{~mL}$ of juice $) ;{ }^{2} \mathrm{Means}$ in the same column, followed by the same letter, are not significantly different (Scott Knott, 5\%); * significant (Scott Knott, 5\%); **= significant (Scott Knott, 1\%) [SS= sólidos solúveis; AT= acidez titulável; VitC= vitamina C; firmeza em Newton; Médias seguidas da mesma letra, na coluna, não diferem entre si (Scott Knott, 5\%); *= significativo (Scott Knott, 5\%); **= significativo (Scott Knott, 1\%)]. 
The solid solubles ( ${ }^{\circ}$ Brix) were of higher values in genotypes Jab-9 $\mathrm{x}$ Jab-7 (13.03), Jab-9 x Jab-3 (11.85), Jab-18 x Jab-9 (11.71), Jab-18 x Jab-20 (11.61), Jab-18 x Jab-11 (11.59), Jab-7 x Jab-9 (11.63), Jab-7 x Jab-18 (11.94), Jab-7 x Jab-20 (12.28), Jab-7 x Jab-3 (11.51), and Bônus $n^{\circ} 2$ (12.27). These values are an indication that the fruits were of very good quality since melon fruit quality is narrowly associated with the content of solid solubles. In a previous research with five melon hybrids, Vargas et al. (2008b) found ${ }^{\circ}$ Brix values between 9.00 and 11.24 , lower, thus, than those found in the present work.

Considering the minimum ${ }^{\circ} \mathrm{Brix}$ $\left(10^{\circ}\right.$ Brix $)$ value acceptable for exportation (Alves , 2000) and with basis on the data here reported, only the genotypes Jab9 x Jab-9, Jab-9 x Jab-20, Jab-18 x Jab-18, Jab-20 x Jab-9, Jab-20 x Jab-20, Jab-20 x Jab-11, Jab-20 x Jab3, Jab-11 x Jab-20, Jab-11 x Jab-11, and Jab-3 x Jab-9 would not reach that value. On the other hand, genotypes Jab-9 $\mathrm{x}$ Jab-7, Jab-7 x Jab-20 and Bônus n 2 are considered of the extra type (Gorgatti Neto et al., 1994).

The highest values of titratable acidity were shown by the genotypes Louis (0.11) and Jab-7 x Jab-9 (0.10). Titratable acidity varied between 0.06 (genotypes Jab-20 x Jab-3 and Jab-3 $\mathrm{x}$ Jab-11) and 0.11 (genotype Louis). Values similar (between 0.09 and 0.13) to these were reported by Vargas et al. (2008b).

Referring to RATIO, which represents the degree of maturation of the fruits, the genotypes showing superior values were Jab-7 x Jab-7, Jab20 x Jab-3, Jab-11 x Jab-7, Jab-3 x Jab-7, and Jab-3 x Jab-3 (Table 2). The high values found for that characteristic are due to the low titratable acidity verified since RATIO is the relation between solid solubles and the titratable acidity.

For the $\mathrm{pH}$ values (6.78 to 7.90 ) and fruit coat thickness ( 1.35 to $2.57 \mathrm{~mm}$ ) no significant differences were observed.
In relation to vitamin $C$, the genotypes Jab-9 x Jab-9, Jab-18 x Jab-11, Jab-7 x Jab-7, Jab-7 x Jab-20, Jab-7 x Jab-11, Jab-20 x Jab-9, Jab$20 \times$ Jab-7, Jab-20 x Jab-20, Jab-20 x Jab-11, Jab-11 x Jab-20, Jab-11 x Jab-11, Jab-3 x Jab-9, and Jab-3 x Jab 20 presented the highest values (Table 2). Melon nutritional quality is highly dependent on its content of Vitamin C (Lester, 1997).

The fruits of the genotypes Jab-7 x Jab-7, Jab-7 x Jab-11, Jab-7 x Jab-3, Jab-11 x Jab-7, Jab-3 x Jab-7, and Louis were those showing the highest degree of firmness. This is the characteristic that imparts resistance to rough handling and transportation to melon fruits. It is noted that the genotypes with the highest values of firmness had the Jab-7 as one of their parental lines.

The minimum mass of $0.8 \mathrm{~kg}$ per fruit of net melon (Gorgatti Neto et al., $1994)$ and the minimum of $10^{\circ} \mathrm{Brix}$ soluble solids (Alves, 2000) allowed to identify the genotypes Jab-9 x Jab-3, Jab-18 x Jab-20, Jab-18 x Jab-11, Jab-7 x Jab-20, Jab-7 x Jab-11, Jab-20 x Jab-7, and, Jab-3 $\mathrm{x}$ Jab-20, as promising. They are therefore suitable for participating in assessment tests in the main net melon producing areas network in Brazil.

\section{ACKNOWLEDGEMENTS}

The authors are grateful to FAPESP (Process 2006/50326-6) and CAPES for supporting this work.

\section{REFERENCES}

ALVES RE. (org) 2000. Melão: pós-colheita. Brasília: Embrapa Comunicação para Transferência de Tecnologia, 43p. (Frutas do Brasil, 10).

CASTELLANE PD; ARAÚJO JAC. 1994. Cultivo sem solo: Hidroponia. Jaboticabal: FUNEP, $43 \mathrm{p}$.

COSTACC; CECÍLIO FILHOAB; CAVARIANNI RL; BARBOSA JC. 2004. Produção do melão rendilhado em função da concentração de potássio na solução nutritiva e do número de frutos por planta. Horticultura Brasileira 22: 23-27.

CRUZ CD. 2006. Programa Genes: Biometria. Editora UFV. Viçosa. 382p.

EMPRESA BRASILEIRA DE PESQUISA AGROPECUARIA - CENTRO NACIONAL DE PESQUISA DE HORTALIÇAS EMPBRAPA-CNPH. 2011, 01 june. Hortaliças em Números. Available at $<$ http://www.cnph. embrapa.br/paginas/hortalicas_em_numeros/ hortalicas_em_numeros.htm>

FOOD AGRICULTURAL ORGANIZATION FAO. 2011, 01 June. Statistical - database. Available at $<$ http://faostat.fao.org/site/339/ default.aspx $>$

GAL S; ALKALAI-TUVIA S; PERTIZELAN Y; ELKIND Y; RAVID U; FALLIK E. 2008. Sensory evaluation of 'Galia' - type melons treated with 1-methylcylopropene after prolonged storage. Journal of Horticultural Science \& Biotechnology 83: 589-594.

GORGATTI NETO A; GAYET JP; BEINROTN EW; MATALLO M; GARCIA EEC; GARCIA AE; ARDITO GFG; BORDIN MR. 1994. Melão para exportação: procedimento de colheita e pós-colheita. Brasília: EmbrapaSPI/Frupex, 37 p. (Frupex, Publicações Técnicas, 6).

INSTITUTO ADOLFO LUTZ. 1985. Normas analíticas do Instituto Adolfo Lutz. Métodos químicos e físicos para análise de alimentos. $3^{\mathrm{a}}$ ed. v.1. São Paulo.

LESTER G. 1997. Melon (Cucumis melo L.) fruit nutritional quality and health funcionality. HortTech 7: 222-227.

PADUAN MT; CAMPOS RP; CLEMENTE E. 2007. Qualidade dos frutos de tipos de melão, produzidos em ambiente protegido. Revista Brasileira de Fruticultura 29: 353-539.

RIZZO AAN; BRAZ LT. 2001. Características de cultivares de melão rendilhado cultivadas em casa de vegetação. Horticultura Brasileira 19: 370-373.

RIZZO AAN; BRAZ LT. 2004. Desempenho de linhagens de melão rendilhado em casa de vegetação. Horticultura Brasileira 22: 784-788.

VARGAS PF; CASTOLDI R; CHARLO HCO; BRAZ LT. 2008a. Desempenho de cultivares de melão rendilhado em função do sistema de cultivo. Horticultura Brasileira 26: 197-201.

VARGAS PF; CASTOLDI R; CHARLO HCO; BRAZ LT. 2008b. Qualidade de melão rendilhado (Cucumis melo L.) em função do sistema de cultivo. Ciência \& Agrotecnologia 32: 137-142.

VARGAS PF; GALATTI FS; SOUZA JO; CHARLO HCO; CASTOLDI R; BRAZ LT. 2010. Avaliação de parentais e híbridos experimentais de melão rendilhado. Ciência \& Agrotecnologia 34: 1102-1108. 